\title{
I. R\&A als Innovation im amerikanischen Nachrichtendienstwesen
}

\author{
Die amerikanischen Nachrichtendienste \\ zu Beginn des Zweiten Weltkrieges
}

$\mathrm{Daß}$ die Vereinigten Staaten noch ein halbes Jahr vor dem japanischen Angriff auf Pearl Harbor nicht über ein zentral koordiniertes Nachrichtendienstwesen, geschweige einen der heutigen Central Intelligence Agency (CIA) vergleichbaren Geheimdienst verfügten, mag wenig in das immer wieder von skandalumwitterten Aktionen ihrer Geheimdienste geprägte Bild der Supermacht USA passen. Tatsächlich hatten jedoch verschiedene Faktoren die Einrichtung eines zentral gelenkten Nachrichten- oder Geheimdienstes lange als politisch unerwünscht und kaum durchsetzbar, wenn nicht gar unnötig erscheinen lassen. Die bis in die Tage George Washingtons zurückreichende Ablehnung von Spionage im engeren Sinne war einer dieser Faktoren; sie verband sich mit der in der amerikanischen Öffentlichkeit bis heute als problematisch empfundenen Frage nach der Vereinbarkeit eines staatlichen Geheimdienstes mit den Idealen einer freiheitlichen und demokratischen Gesellschaft zu einem schwer zu entkräftenden Argument gegen die Einrichtung einer solchen Organisation. Solange die Vereinigten Staaten sich im Zeichen des Isolationismus hinter die Ozeane zurückzuziehen suchten, schien überdies gar keine Notwendigkeit für die Etablierung eines Geheimdienstes gegeben ${ }^{1}$.

Vor diesem Hintergrund konnten die schließlich durchaus bestehenden, wenn auch meist in einem wörtlich zu nehmenden Sinne mit nachrichtendienstlichen Aufgaben betrauten Abteilungen von Army, Navy, FBI und State Department sowie einiger anderer Regierungsbehörden als ausreichend gelten, um die innere wie äußere Sicherheit der Vereinigten Staaten zu garantieren und die politischen Entscheidungsträger mit Daten und Informationen zu versorgen ${ }^{2}$. Schon letzteres fiel freilich nicht immer zur Zufriedenheit der betreffenden Politiker aus, da die Nachrichtendienststellen der verschiedenen Institutionen im allgemeinen nicht zusammenarbeiteten, sondern vielmehr dazu tendierten, die im Rahmen ihrer jeweiligen Kompetenzen gesammelten Daten mit einer den Auffassungen ihrer Behörde entsprechenden Interpretati-

${ }^{1}$ Die verschiedenen Tendenzen und politischen Konstellationen, die dem Aufbau eines zentralen Nachrichtendienstwesens entgegenstanden, sind nirgendwo zusammenhängend dargestellt, werden in der einschlägigen Literatur aber im jeweils relevanten Kontext immer wieder angesprochen, vgl. Troy, Donovan, S. 3 ff. - eine 1975 fertiggestellte, ursprünglich für den CIA-internen Gebrauch bestimmte Studie, die, zunächst als "Secret“ klassifiziert, 1981 freigegeben und in nur leicht abgeänderter Form veröffentlicht wurde; Cline, Secrets, S. 1 ff.; Ransom, Secret Intelligence in the United States, 1947-1982, in: Andrew/Dilks (Hrsg.), Missing Dimension, S.199226; sowie Jeffreys-Jones, The CIA and American Democracy, S.11ff.

${ }^{2}$ Vgl. die graphische Darstellung bei Cline, Secrets, S. 8. Zu Geschichte und Entwicklung des amerikanischen Nachrichtendienstwesens bis zum Ende der zwanziger Jahre Troy, Donovan, S.3-11; Corson, Armies of Ignorance, S.41-75; sowie den Überblick bei Ameringer, U.S. Foreign Intelligence, S. 17-127. 
on weiterzugeben ${ }^{3}$. Die Empfänger der so ausgearbeiteten Studien und Berichte sahen sich deshalb nicht selten mit widersprüchlichen Informationen und Empfehlungen konfrontiert, aus denen nur mühsam eine Grundlage für verantwortliche Entscheidungen zu erarbeiten war.

Das Fehlen jeglicher koordinierter Nachrichtendiensttätigkeit sollte in der zunehmend gespannten weltpolitischen Situation der dreißiger Jahre immer problematischer werden, und angesichts des Kriegsausbruchs in Europa wurde diese Situation vollends unhaltbar. Seit 1939 gab es zwar Bestrebungen, die Arbeit der bestehenden Nachrichtendienststellen zu koordinieren, und man hatte mit der Einrichtung von Secret-Intelligence-Abteilungen im FBI und in der Marine zudem begonnen, den bislang stark vernachlässigten Bereich der Auslandsspionage auszubauen. Im Hinblick auf die Leistungsfähigkeit des amerikanischen Nachrichtendienstwesens waren dennoch kaum substantielle Verbesserungen zu verzeichnen ${ }^{4}$. Präsident Roosevelt, der vielleicht nicht zu Unrecht als "greatest victim ${ }^{* 5}$ der unzulänglichen Arbeit der Nachrichtendienste bezeichnet worden ist, versuchte schließlich, sich in der ihm eigenen unbürokratischen Weise zu behelfen, indem er den Journalisten John Franklin Carter (alias Jay Franklin) beauftragte, zusammen mit qualifizierten Mitarbeitern Berichte und Analysen zu aktuellen Problemen auszuarbeiten ${ }^{6}$. Daß dieser „Privatgeheimdienst“ kaum mehr als ein Provisorium darstellte, mag dem Präsidenten zwar bewußt gewesen sein, doch dürfte er auch kaum Zweifel daran gehegt haben, daß jede umfassendere Lösung auf den erbitterten Widerstand der bestehenden Nachrichtendienststellen stoßen würde, die hinter jedem Reformvorschlag eine Einschränkung ihres Kompetenz- und Einflußbereiches witterten. Die im Rahmen der sich seit Anfang 1941 ausbildenden anglo-amerikanischen Koalition erforderlich werdende Zusammenarbeit zwischen den Geheimdienststellen beider Länder ließ allerdings eine bessere Koordinierung der amerikanischen Nachrichtendiensttätigkeit immer dringlicher erscheinen, doch zeigten sich die bislang nicht an Kooperation gewöhnten US-Nachrichtendienste unfähig, die notwen-

${ }^{3}$ Insofern ist die Verwendung des im Englischen und Amerikanischen gängigen Begriffs „intelligence community" nicht unproblematisch; vgl. dazu Winks' Kritik an dieser punduly cozy phrase", in: ders., Cloak, S. 62, sowie die Einleitung der Herausgeber in Andrew/Dilks (Hrsg.), Missing Dimension, S. 1-16.

${ }^{4}$ Eine scharfzüngige Kritik an der Arbeit der bestehenden US-Nachrichtendienststellen findet sich in NA, RG 226, E 99, b 76, f 45/46: History of the Research and Analysis Branch in the Office of Strategic Services, June 1941 - September 1944 (künftig zitiert als R\&A-History). Zur Entwicklung des amerikanischen Nachrichtendienstwesens in der zweiten Hälfte der dreiBiger Jahre Troy, Donovan, S.11-21; Corson, Armies of Ignorance, S.77-107; Cline, Secrets, S. $3 \mathrm{ff}$., sowie die abweichende Darstellung bei Jeffreys-Jones, American Espionage, S.120ff., $133 \mathrm{ff}$.

${ }^{5}$ OSS War Report I, S.7. Das Manuskript zu dieser offiziellen Darstellung der Entwicklung von COI/OSS befindet sich in NA, RG 226. Bereits im Juli 1944 war ein "OSS History Project“ ins Leben gerufen worden (NA, RG 226, E 1, b 17, f: OSS History Project, Special Order vom 25.7. 1944); die in diesem Rahmen ausgearbeiteten Manuskripte - darunter auch die für diese Arbeit herangezogene R\&A-History - waren z.T. sehr umfänglich. Die Auflösung des OSS nach Kriegsende erlaubte jedoch lediglich die Fertigstellung des relativ knappen OSS War Report, der, ursprünglich als "Top Secret" klassifiziert, nach Ablauf der dreißigjährigen Sperrfrist 1976 in kaum überarbeiteter Form veröffentlicht wurde.

${ }^{6}$ Smith, Shadow Warriors, S.63 f. Die von Carter für Roosevelt ausgearbeiteten Berichte befinden sich in FDRL, PSF/Subject File, b 122-125, 126-139 (vgl. b 208-219). 
digen Maßnahmen durchzuführen ${ }^{7}$. Letztlich trugen sie damit zu einer Entwicklung bei, die von ihnen am wenigsten begrüßt wurde, denn ihr Versagen ermöglichte es einem Außenseiter wie dem etwas exzentrischen New Yorker Anwalt William J. Donovan, die Vorstellungen zu verwirklichen, die er selbst teils aus privatem Interesse, teils im Rahmen zweier Englandreisen als Sonderbeauftragter der amerikanischen Regierung zu einer Verbesserung des US-Nachrichtendienstwesens entwickelt hatte.

Donovan gehörte zu jenem „old boys' network“, das sich aus ehemaligen Regierungsbeamten und Militärs sowie Geschäftsleuten konstituierte, die sich "for the love of the thing" - also ohne Bezahlung oder offizielles Amt - ihrer Regierung hin und wieder als Amateuragenten zur Verfügung stellten ${ }^{8}$. In dieser Eigenschaft war Donovan seit Sommer 1940 bereits zweimal tätig gewesen, wobei er Gelegenheit gehabt hatte, sich mit den Mängeln des amerikanischen Nachrichtendienstwesens gründlich vertraut zu machen. Im Juli 1940 war er auf Anregung des mit ihm befreundeten Marineministers Frank Knox in geheimer Mission nach London gesandt worden, um zu erkunden, ob Großbritannien mit amerikanischer Unterstützung eine realistische Chance hätte, den deutschen Angriffen standzuhalten, oder ob es - wie eben erst Frankreich - innerhalb kürzester Zeit zusammenbrechen würde.

Donovan mochte aufgrund seiner Persönlichkeit und seiner beruflichen Erfahrungen ${ }^{9}$ als geeignet gelten, einem so komplexen wie politisch brisanten Auftrag gewachsen zu sein. Obwohl ihm wenig Erfolg beschieden gewesen war, als er sich, hochdekoriert aus dem Ersten Weltkrieg zurückgekehrt, um eine Karriere als republikanischer Politiker bemühte, trauten ihm Freunde und Bekannte in diesem Bereich stets Großes zu, und selbst Roosevelt soll einmal gemeint haben, „if Bill Donovan had been a Democrat, he'd been in my place today “10. Tatsächlich war Donovan in der politischen Arena jedoch ein glückloser Kandidat und verlor sämtliche Wahlen, denen er sich je stellte. Im Gegensatz dazu gestaltete sich seine juristische Karriere überaus erfolgreich. Anfang der dreißiger Jahre hatte Donovan in New York ein Anwaltsbüro begründet, das bald zu den renommiertesten des Landes gehörte. Er und seine Mitarbeiter spezialisierten sich auf die komplizierten Rechtsstreitigkeiten amerikanischer Großkonzerne, und wenn Donovan später das systematische Sammeln und Auswerten von Hintergrundinformationen und aktuellen Daten als unverzichtbare Grundlage für politische Entscheidungen und strategische Planungen bezeichnete, konnte er immerhin darauf verweisen, daß Erfolg und Renommee von „Donovan Leisure Newton \& Irvine" nicht zuletzt auf der Anwendung dieser Methode beruhten ${ }^{11}$.

7 Einzelheiten zu den im Frühjahr 1941 unternommenen Reformversuchen des US-Nachrichtendienstwesens bei Troy, Donovan, S.43-51; vgl. auch Cline, Secrets, S.1-30, sowie Smith, Shadow Warriors, S. $57 \mathrm{ff}$.

${ }^{8}$ Dazu Corson, Armies of Ignorance, S. $73 \mathrm{ff}$.; Smith, Shadow Warriors, S.27; Andrew/Dilks (Hrsg.), Missing Dimension, S.7ff.; Knightley, Geschichte der Spionage im 20. Jahrhundert, S.201f.

9 Es gibt mehrere Donovan-Biographien, die allerdings zumindest mit Blick auf Donovans Tätigkeit als COI- bzw. OSS-Direktor nicht immer zuverlässig sind; fast schon hagiographisch Ford, Donovan of OSS, und Dunlop, Donovan, America's Master Spy; kritischer Cave Brown, The Last Hero; umfassende und verläßliche Darstellung von Donovans Tätigkeit als COI/OSS-Direktor bei Troy, Donovan.

${ }^{10}$ Ebenda, S. 26.

11 Ebenda, S. $26 \mathrm{f}$. 
Das Sammeln und Auswerten von Informationen war für Donovan aber nicht nur ein aus seiner beruflichen Tätigkeit resultierendes Erfordernis, sondern auch privates Steckenpferd. Seine zahlreichen Reisen nach Übersee, ob geschäftlicher oder privater Natur, waren im Grunde stets „fact-finding missions“. So hatte er 1919 seine verspätete Hochzeitsreise nach Japan unterbrochen, um auf Drängen des dortigen amerikanischen Botschafters in Sibirien Nachforschungen über das Regime Admirals Alexander Koltschaks anzustellen. Auf zahllosen Europareisen betätigte sich Donovan in ähnlicher Weise; so etwa 1935, als er auf eigene Initiative, aber in Absprache mit dem War Department, Hintergründe und Entwicklung der Äthiopienkrise vor Ort recherchierte und dabei mit hohen Militärs und führenden Politikern sowie dem „Duce“ selbst zusammentraf. Auch in der zweiten Hälfte der dreißiger Jahre unternahm Donovan zahlreiche ähnliche Reisen, so daß er für den ihm im Sommer 1940 erteilten Auftrag in vielerlei Hinsicht bestens vorbereitet war: Er verfügte nicht nur über eine umfassende Kenntnis der politischen und militärischen Entwicklungen in Europa, sondern auch über zahlreiche wertvolle Kontakte - kurzum: „he knew, or had a friend who knew, nearly everyone who counted "12.

Insgesamt sollte sich Donovan seines Auftrages dann auch in so umfassender und umsichtiger Weise entledigen, daß gesagt worden ist, er habe dabei seine künftige Funktion als „Coordinator of Information“ schon vorweggenommen, und tatsächlich hat er selbst die Anfänge des von ihm begründeten und geleiteten Nachrichtendienstes später auf diese Reise zurückgeführt ${ }^{13}$. In London mag Donovan auch ermuntert worden sein, sich für eine Verbesserung des amerikanischen Nachrichtendienstwesens einzusetzen, denn da die Briten hofften, in den Vereinigten Staaten bald einen Partner im Kampf gegen Hitler zu haben, mußte ihnen daran gelegen sein, daß die USA über ein effizientes Nachrichtensystem bzw. einen Geheimdienst verfügten, der mit den entsprechenden britischen Stellen zusammenarbeiten würde. Bereits im Frühjahr 1940 hatte der kanadische Millionär William S. Stephenson seine weitreichenden Verbindungen genutzt, um eine Zusammenarbeit zwischen dem britischen Secret Intelligence Service (SIS) und dem amerikanischen FBI in die Wege zu leiten, und als Stephenson im Sommer 1940 den Posten des „British Security Coordinator“ in New York übernahm, gehörte neben seinem vorrangigen Auftrag, sich für ausreichende Hilfeleistung an Großbritannien einzusetzen, die Förderung einer anglo-amerikanischen Kooperation in Sachen Geheimdienst zu seinen wichtigsten Aufgaben ${ }^{14}$.

$\mathrm{Zu}$ den zahlreichen Personen, mit denen Stephenson Umgang pflegte, um diese Ziele zu erreichen, gehörte auch der entschieden für die Unterstützung Großbritanniens eintretende Donovan. Stephensons Bemühungen, die verantwortlichen Stellen in Wash-

\footnotetext{
12 Winks, Cloak, S. 65.

13 Troy, Donovan, S. 33, 53.

14 Hyde, The Quiet Canadian, S. $25 \mathrm{ff} ., 52 \mathrm{ff}$.; die von dem fast namensgleichen William Stevenson verfaßte Biographie Stephensons ist nicht immer zuverlässig: Stevenson, A Man Called Intrepid. Kritisch dazu Stafford, ,Intrepid': Myth and Reality, in: JCH 22/2 (1987), S. 303-317. Vgl. außerdem: Troy, Donovan, S.34f.; Smith, Shadow Warriors, S. 29 f.; Corson, Armies of Ignorance, S. 108 f., sowie die überblickshafte Darstellung von Stephenson selbst in Hyde, Secret Intelligence Agent, S. $247 \mathrm{ff}$. Zum britischen Interesse an der Einrichtung eines US-Nachrichtendienstes Troy, Donovan, S.54f., $59 \mathrm{ff}$.; Cline, Secrets, veranschlagt die britische Einflußnahme sehr hoch, vgl. besonders S. $21 \mathrm{ff}$., $36 \mathrm{f}$.
} 
ington zu überzeugen, daß ein Ausbau des US-Nachrichtendienstwesens dringend notwendig sei ${ }^{15}$, wurden von Donovan zumindest insofern unterstützt, als dieser Ende November 1940 anläßlich eines Vortrags über die Kriegsbereitschaft der USA die Bildung eines Ausschusses vorschlug, der aktuelle politische Probleme anhand verfügbarer Informationen studieren sollte, um umfassende Berichte und Analysen zu erarbeiten, die der Administration als Entscheidungsgrundlage dienen konnten. Damit skizzierte Donovan im Grunde schon die von ihm später als so wesentlich bezeichnete Forschungs- und Analysefunktion eines Nachrichtendienstes ${ }^{16}$. Bevor er aber in dieser Hinsicht weiter aktiv werden konnte - falls er dies Ende 1940 überhaupt schon beabsichtigte $^{17}$-, hatte Donovan einmal mehr Gelegenheit, als „Ein-Mann-Geheimdienst“ zu fungieren.

Inzwischen hatte sich nämlich herausgestellt, daß Großbritannien über die von den USA gewährte materielle Unterstützung hinaus auch auf Begleitschutz für britische Handelsschiffe bei der Überquerung des Atlantik angewiesen war. Die verantwortlichen Politiker in Washington standen so erneut vor der Frage, ob die militärische, wirtschaftliche und politische Verfassung Großbritanniens eine noch weitergehende amerikanische Verwicklung rechtfertigte. Da es in Washington einmal mehr an zuverlässigen Informationen und Analysen zu dieser Frage fehlte, bot sich Donovan an, eine zweite Inspektionsreise nach Großbritannien zu unternehmen und im Anschluß an seinen dortigen Aufenthalt auch die Krisengebiete im Mittelmeerraum und auf dem Balkan zu bereisen. Dieser Vorschlag fand nicht nur die notwendige Billigung durch den Präsidenten und die Secretaries of State, War und Navy, sondern löste in London sogar so etwas wie Enthusiasmus aus - immerhin betrachtete der britische Botschafter in Washington Donovan inzwischen als "one of our best and most influential friends here “18.

In Begleitung von Stephenson trat Donovan am 6. Dezember 1940 seine zweite Mission als Sonderbeauftragter der amerikanischen Regierung an. Wie schon bei seinem ersten Aufenthalt im Sommer traf Donovan in der britischen Hauptstadt wiederum auf großes Entgegenkommen, und seine sich an den Aufenthalt in London anschließende ausgedehnte Inspektionsreise in die Balkan- und Mittelmeerländer wurde von den Briten nicht nur finanziert, sondern auch in vielerlei anderer Hinsicht erleichtert ${ }^{19}$. Als

15 Troy, Donovan, S. 54; zum Verhältnis zwischen Donovan und Stephenson vgl. ebenda, S. 34 ff., $53 \mathrm{ff}$;; sowie Stephenson in Hyde, a.a.O.; Knightley, Geschichte der Spionage, S.205f., sieht Stephenson gar als Drahtzieher hinter Donovans Englandreisen und Donovan als „Opfer eines Manövers, das man nur als intensive PR-Kampagne bezeichnen kann und das einer der seltenen Triumphe des britischen Geheimdienstes im Zweiten Weltkrieg war ${ }^{\text {". }}$.

16 Vgl. dazu Troy, Donovan, S.29. Donovan mag dabei eine gewissermaßen offizielle Variante des seit 1921 aktiven Council on Foreign Relations vorgeschwebt haben; zu Zusammensetzung und Aktivitäten des Council vgl. Wala, Winning the Peace.

17 Donovans Engagement für eine Verbesserung des amerikanischen Nachrichtendienstwesens bzw. seine Pläne für die Schaffung eines zentralen US-Geheimdienstes sind in der Literatur häufig "vordatiert" worden; kritisch dazu Troy, Donovan, S. $52 \mathrm{ff}$.

${ }^{18}$ Zit. nach Troy, Donovan, S.37; die genauen Hintergründe von Donovans zweiter Reise nach Großbritannien sind nicht völlig geklärt, vgl. ebenda, S.36ff.; Smith, Shadow Warriors, S. $40 \mathrm{ff}$; Corson, Armies of Ignorance, S. $123 \mathrm{ff}$.

19 Dies geschah freilich nicht aus uneigennützigen Motiven; besonders Churchill scheint gehofft zu haben, daß Donovan in den Balkanländern als Fürsprecher britischer Interessen auftreten und versuchen würde, die Regierungen dieser Staaten für ein entschiedenes Auftreten gegen 
Donovan nach fast drei Monaten Anfang März 1941 nach Washington zurückkehrte, dürfte er vom Krieg tatsächlich mehr gesehen haben "than any other prominent figure in American public life" 20 . Aber nicht nur den europäischen Krieg hatte Donovan eingehender in Augenschein nehmen können als andere prominente Amerikaner - auch in die Arbeit des britischen Geheimdienstes hatte man ihm mehr Einblick gegeben, als einem Nicht-Briten wohl je zuvor und vielleicht auch jemals seither (freiwillig) gewährt worden ist ${ }^{21}$.

Während Donovan dies vor allem Stephenson zu verdanken gehabt haben dürfte, lagen dem scheinbaren Leichtsinn, mit dem die Briten einen amerikanischen Zivilisten ohne offizielles Amt in kriegswichtige und damit geheimste Erkenntnisse einweihten, auch ganz handfeste Motive zugrunde: Zum einen konnten es sich die Briten Ende 1940/Anfang 1941 angesichts ihrer relativ schwachen militärischen Position kaum erlauben, gegenüber einem Abgesandten Präsident Roosevelts, der - wie wohlgesonnen auch immer - schließlich gekommen war, ihre Chancen im Kampf gegen Hitler einzuschätzen, einen „Joker“ im Ärmel zu behalten. Zwar beschränkten sich die einzig wirklich eindrucksvollen Erfolge des britischen Geheimdienstes Ende 1940 auf die ersten im Rahmen von ULTRA gewonnenen Erkenntnisse - und Donovan ist über dieses Projekt wohl nur indirekt informiert worden ${ }^{22}$. Doch war Donovan seit seiner eigenen Kampagne gegen die nationalsozialistische „Fünfte Kolonne“ im Herbst $1940^{23}$ genügend von der Bedeutung subversiver Aktionen und psychologischer Kriegführung überzeugt, um durch die noch recht bescheidenen Anfänge der britischen Special Operations Executive (SOE) beeindruckt zu $\operatorname{sein}^{24}$. Zudem mag vor allem Stephenson gehofft haben, daß Donovan, der seit Kriegsbeginn vielleicht tatsächlich ein wenig na hero in search of a role ${ }^{25}$ war, sich letztlich doch noch des amerikanischen Nachrichtendienstwesens annehmen und dabei - zum Nutzen der Briten - ihre Organisation als Vorbild sehen würde.

Diese Hoffnungen schienen sich zu erfüllen, als Donovan am Tag nach seiner Rückkehr dem Präsidenten einen ersten Bericht über seine Reise erstattete, denn es ist be-

Hitlerdeutschland zu gewinnen. Später sind die politischen Folgen von Donovans Unterredungen im Negativen wie Positiven zuweilen überschätzt worden, vgl. z. B. Ford, Donovan, S.99106; sehr kritisch, doch insgesamt nüchtern abwägend Smith, Shadow Warriors, S. $44 \mathrm{ff} ., 51 \mathrm{f}$.; umfassende Darstellung der britischen Politik in den Balkanländern während des Zweiten Weltkriegs bei Barker, British Policy in South-East Europe, zu Donovans Mission siehe S. 101, 119. Einzelheiten zu Donovans Reiseroute bei Troy, Donovan, S.37ff.; Smith, Shadow Warriors, S. $47 \mathrm{ff}$.; Corson, Armies of Ignorance, S. $127 \mathrm{ff}$.

${ }^{20}$ Smith, Shadow Warriors, S. 54.

21 Corson, Armies of Ignorance, S. 129; Troy, Donovan, S. 39.

${ }^{22} \mathrm{Zu}$ ULTRA, der Entzifferung des deutschen Codes durch den britischen Geheimdienst, siehe Winterbotham, Aktion Ultra; zur Verfügbarkeit von ULTRA Lewin, Entschied Ultra den Krieg?

23 Troy, Donovan, S. 33, weist darauf hin, daß Donovan den Journalisten Edgar Ansel Mowrer bei dessen „Aufklärungskampagne“ über die Aktivitäten der „Fünften Kolonne“ unterstützte, vgl. William J. Donovan, Edgar Mowrer, Fifth Column Lessons for America, Washington, D. C., 1941.

${ }^{24}$ Die Situation des britischen SOE und SIS Ende 1940 ist skizziert bei Smith, Shadow Warriors, S. 42 ff.; eine ausgezeichnete Darstellung der Geschichte der SOE bietet Stafford, Britain and the European Resistance 1940-1945.

${ }^{25}$ Troy, Donovan, S. 52. 
hauptet worden, daß er bei dieser Gelegenheit "the creation of a new agency“ vorgeschlagen habe ${ }^{26}$. Diese „new agency“ sollte mit nachrichtendienstlichen Aufgaben betraut werden und zudem für Propaganda, psychologische Kriegführung, Sabotage und Guerillakampf zuständig sein. Während nicht geklärt ist, ob Donovan diesen Vorschlag wirklich schon während jener Unterredung mit Roosevelt erwähnt hat, setzte er sich unzweifelhaft bald nach seiner Rückkehr nach Washington für die Schaffung eines zentralen US-Geheimdienstes ein, denn Brigadegeneral Sherman Miles, stellvertretender Stabschef für den Nachrichtendienst G-2, äußerte sich am 8. April 1941 in einem Schreiben an Stabschef General George C. Marshall besorgt über „a movement [...] fostered by Col. Donovan, to establish a super agency controlling all intelligence ${ }^{\text {"27. }}$.

Eine erste schriftliche Äußerung Donovans zu diesem Thema liegt allerdings erst vom 26. April 1941 vor $^{28}$ - und Miles' Befürchtungen lassen sich aus diesem Schriftstück nur bedingt rechtfertigen. Auf Anregung von Knox skizzierte Donovan in einem Schreiben an diesen kurz die Organisation der britischen Auslandsnachrichtendienste, was er ganz offensichtlich mit Blick auf die Möglichkeit tat, daß ein ähnliches Instrumentarium auch bald in den Vereinigten Staaten geschaffen werden könnte. In diesem Zusammenhang betonte Donovan aber ausdrücklich, daß eine derartige Organisation in keiner Weise die "home duties“ der Nachrichtendienststellen von Armee und Marine sowie des FBI beschränken oder gar übernehmen sollte. Donovan schwebte ein reiner Auslandsnachrichtendienst vor; dieser sollte allerdings auch alle verfügbaren Informationen für den Präsidenten bzw. für von ihm autorisierte Dienststellen auswerten, was implizierte, daß die neue Behörde als Zentrale dienen sollte, an die von den bereits bestehenden Nachrichtendiensten gesammelte Informationen weitergegeben würden, um zu Berichten und Analysen für die Administration verarbeitet zu werden. Damit nahm Donovan in modifizierter Form seine schon früher formulierte Vorstellung eines Organs auf, das im wesentlichen eine Forschungs- und Analysefunktion ausüben sollte. Das Sammeln und Auswerten von Informationen bezeichnete Donovan in diesem Schreiben an Knox als "intelligence work“ im engeren Sinne; weitere wichtige Aufgabenbereiche sah er in der Auswertung von Post, im Abhören von Funkverkehr, im Einsatz von Propaganda als einem Mittel psychologischer Kriegführung und in der Planung und Ausführung subversiver Aktionen. Diese Auflistung dürfte vor allem die Aspekte geheimdienstlicher Tätigkeit widerspiegeln, auf deren Bedeutung Donovan während seiner beiden Reisen von den Briten immer wieder aufmerksam gemacht worden war; zudem ist nicht auszuschließen, daß Stephenson einen Anteil an der Formulierung dieses Schreibens an Knox hatte.

Am 10. Juni 1941 legte Donovan dann mit seinem „Memorandum of Establishment of Service of Strategic Information " ${ }^{29}$ eine formelle Empfehlung für die Einrichtung eines zentralen US-Geheimdienstes vor. Die in dieser Denkschrift formulierten Überlegungen können kaum als ein in sich schlüssiges Konzept bezeichnet werden ${ }^{30}$, son-

\footnotetext{
${ }^{26}$ Wallace R. Deuel in einem Entwurf zu einer "History of the OSS“, zit. nach Troy, Donovan, S. 40.

27 Ebenda, S. 42.

${ }^{28}$ In Auszügen abgedruckt ebenda, S. $417 \mathrm{f}$.

29 Abgedruckt ebenda, S.419f., sowie bei Corson, Armies of Ignorance, S. $135 \mathrm{ff}$.

${ }^{30}$ Besonders kritisch Smith, Shadow Warriors, S. $64 \mathrm{ff}$.
} 
dern stellen eher ein Konglomerat aus den vielerlei Anregungen dar, die Donovan auf seinen Reisen, während seiner Gespräche mit Stephenson und wahrscheinlich auch durch Diskussionen in Washington erhalten hatte. Donovan begann sein Memorandum mit einigen grundsätzlichen Überlegungen zum modernen Krieg, der als "totaler Krieg " die Mobilisierung aller Ressourcen einer Nation notwendig mache. Von entscheidender Bedeutung sei deshalb die Fähigkeit, aufgrund möglichst korrekter Analysen der Gesamtstärke des Gegners dessen Absichten und Planungen vorauszusehen, um sie durch die eigene Strategie zu vereiteln. Donovan kritisierte, daß die dazu notwendige Information der amerikanischen Regierung und ihren Planern nicht zur Verfügung stehe, da die Nachrichtendienstabteilungen von Armee und Marine zu sehr auf die Sammlung aktueller technischer bzw. strategischer Informationen spezialisiert seien. Überdies konnte das Sammeln von Daten und Informationen nach Donovans Ansicht nicht genügen; er argumentierte, daß erst ihre Analyse und Interpretation durch Experten aus allen Bereichen der Wissenschaft und Technik zu den Aufschlüssen verhelfe, die im modernen Krieg von so entscheidender Bedeutung seien.

Wie bereits angedeutet, hatte Donovan ähnliche Überlegungen im Ansatz schon früher entwickelt. In seinem Memorandum vom 10.Juni 1941 sind sie jedoch detaillierter ausgeführt. Erstmals formuliert ist hier auch der Gedanke, Experten zur Auswertung der gesammelten Daten und Informationen heranzuziehen, was letztlich eine Verwissenschaftlichung bzw. Professionalisierung von Nachrichtendienstarbeit bedeutete und mithin sehr modern gedacht war ${ }^{31}$. Die Analyse und Interpretation aller eingehenden Informationen erscheint zudem in diesem Rohentwurf eines "Service of Strategic Information" als zentrale Aufgabe dieser Organisation, und unmißverständlich führen Donovans diesbezügliche Überlegungen zur „Research and Analysis“-Funktion des einen Monat später eingesetzten "Coordinator of Information“. Daß Donovan „Research and Analysis" als unverzichtbare Grundlage für geheimdienstliche Operationen betrachtete, wird auch in seinen Vorstellungen zur psychologischen Kriegführung deutlich, wenn er argumentiert, daß zwar die Deutschen bislang Meister im Einsatz der in diesem Bereich wichtigsten "Waffe", dem Radio, seien, daß aber die Wirkung von Radiopropaganda durchaus noch perfektioniert werden könne - „[but] only by planning, and planning is dependent upon accurate information“, wobei „accurate information" in Donovans Verständnis nie nackte Fakten, sondern immer verarbeitete, in die relevanten Zusammenhänge eingeordnete Daten bedeutete.

Mit den Aufgabenbereichen "Research and Analysis“ und Radiopropaganda skizzierte Donovan in seinem Memorandum die beiden Funktionen, die seiner Ansicht nach integrale Bestandteile eines modernen Nachrichtendienstes zu sein hatten, im Rahmen des bestehenden US-Nachrichtenwesens aber nicht abgedeckt waren. Seine Versicherung, "that the proposed centralized unit will neither displace nor encroach upon the FBI, Army and Navy Intelligence, or any other department of the govern-

${ }^{31}$ Die Aktualität der entsprechenden Überlegungen und Vorschläge Donovans wird deutlich in Laqueur, World of Secrets, wo sowohl OSS wie R\&A allerdings nur beiläufig erwähnt sind, so daß bei den für die "Future of Intelligence“ (S.311) gegebenen Empfehlungen nicht berücksichtigt wird, daß bereits in der Vergangenheit versucht wurde, manche von Laqueurs Forderungen zu realisieren. Vgl. in diesem Zusammenhang auch Ameringer, U.S. Foreign Intelligence, S. $396 \mathrm{ff}$., 405. Jeffreys-Jones, American Espionage, S.173ff., bestreitet dagegen den innovativen Charakter von Donovans Konzeptionen. 
ment", hätte insofern glaubwürdig und beruhigend für die angesprochenen Stellen klingen können. Doch wie kaum anders zu erwarten, tendierte man in militärischen Kreisen dazu, die von General Miles schon früh geäußerte Besorgnis zu teilen, daß es Donovan darum ginge, „to establish a super agency controlling all intelligence“. Andererseits fanden Donovans Vorschläge aber auch einflußreiche Befürworter, und neben seinen persönlichen und politischen Freunden wie etwa Frank Knox setzten sich vor allem die Briten für die Verwirklichung seiner Ideen ein. Zudem konnte sich Donovan sicher sein, mit seinem Memorandum auf ernsthaftes Interesse bei Roosevelt zu treffen. Nach einer Unterredung mit Donovan autorisierte der Präsident denn auch am 18. Juni das Bureau of the Budget, einen Entwurf für die Einrichtung des von Donovan vorgeschlagenen „Service of Strategic Information“ auszuarbeiten.

Letztlich sollten allerdings mehrere Entwürfe notwendig sein, bis die Bedenken aller an der Diskussion beteiligten Stellen soweit ausgeräumt waren, daß dem Präsidenten das gewünschte Dokument zur Unterzeichnung vorgelegt werden konnte ${ }^{32}$. Die von den Juristen des Budget Bureau ausgehandelte Kompromißformel vermied - ganz im Gegensatz zu Donovans Memorandum und Roosevelts ursprünglichen Anweisungen - auf Insistieren von Kriegsminister Stimson jeglichen Hinweis auf etwaige militärische Funktionen der neuen Behörde. So wurde Donovan als deren Leiter nicht, wie ursprünglich vorgesehen, zum Generalmajor befördert, und aus seinem „Service of Strategic Information " wurde in eigentümlicher Verbindung zwischen dem Direktor und der ihm unterstellten Organisation ein "Coordinator of Information" (COI). Die Hauptaufgabe der neuen Behörde sollte darin bestehen, „to collect and analyze all information and data, which may bear upon national security; to correlate such information and data, and to make such information and data available to the President and to such departments and officials of the Government as the President may determine".

Damit war die von Donovan als so wichtig erachtete Research and Analysis-Funktion umschrieben; sie blieb die einzige Aufgabe, die die präsidentielle Order der neuen Behörde ausdrücklich zuwies. Darüber hinaus war der COI lediglich noch autorisiert „to carry out, when requested by the President, such supplementary activities as may facilitate the securing of information important for national security not now available to the Government" - eine wachsweiche Formulierung, hinter der sich, freilich nicht ohne Absicht, alles und nichts verbergen konnte. Bezeichnenderweise war in dieser so vage gehaltenen und nur etwa eine halbe Schreibmaschinenseite beanspruchenden Order aber Raum, noch einmal ausdrücklich und ausführlich darauf hinzuweisen, daß die Kompetenzen der regulären militärischen Berater des Präsidenten durch die neue Behörde in keiner Weise beschnitten würden.

Der bürokratische Grabenkampf, der sich schon an der Abfassung dieses Dokuments entzündet hatte, sollte Donovans Behörde zwar von Anfang an das Dasein erschweren und sie bald gar in ihrem Bestehen gefährden, doch war Donovan schließlich kein Neuling in Washington und zudem nicht leicht zu entmutigen. Als Roosevelt am 11.Juli 1941 Donovans Einsetzung als Coordinator of Information durch seine Unterschrift amtlich machte, konnte dieser immerhin für sich verbuchen, gegen große Wi-

32 Einzelheiten bei Troy, Donovan, S.62-70; die vom Präsidenten unterzeichnete Designation eines "Coordinator of Information“ ist abgedruckt ebenda, S.423; vgl. auch Corson, Armies of Ignorance, S. 137 ff.; sowie Smith, Shadow Warriors, S. $65 \mathrm{ff}$. 
derstände die Einrichtung des ersten zentralen Geheimdienstes in der Geschichte der Vereinigten Staaten initiiert zu haben. Aufbau und Entwicklung dieses Geheimdienstes sollte er im folgenden stark prägen, oft genug ist er denn auch als "Vater" des COI bzw. des aus diesem hervorgehenden Office of Strategic Services (OSS) bezeichnet worden. Doch auch wenn die Bedeutung von Donovans Engagement nicht unterschätzt werden sollte, wäre die Schaffung eines zentralen US-Geheimdienstes ohne die durch den Krieg diktierten Erfordernisse wohl noch lange undenkbar geblieben, und sosehr Donovan Entwicklung und Aufbau von COI und OSS auch geprägt haben mag, sosehr prägten eben von Anfang an auch kriegsbedingte Anforderungen beide Organisationen.

\section{Der Aufbau der R\&A-Branch}

Als Donovan am 11.Juli 1941 das Amt des Coordinator of Information antrat, übernahm er damit die Leitung einer Behörde, die noch gar nicht existierte; für deren Organisation - außer den von ihm selbst in seinem Memorandum vom 10. Juni 1941 entwikkelten Vorstellungen - keinerlei Konzeptionen vorlagen, für deren Unterbringung kein Raum und für deren Aufbau und Unterhalt keine Mittel eingeplant waren. Da die Einrichtung eines zentralen US-Nachrichtendienstes im Juli 1941 längst überfällig war, blieb auch keine Zeit, Konzeptionen zu entwickeln und entsprechende Planungen und Vorbereitungen in die Wege zu leiten. Aufbau und Organisation dieses ersten zentralen US-Nachrichtendienstes wurden so unvermeidlich zu einem „learn as you go“Prozeß, der nicht selten nach dem Muster von "trial and error" ablief. An der Frage, ob der COI bzw. das später aus ihm hervorgehende Office of Strategic Services dabei insgesamt mehr Fehlschläge oder mehr Erfolge zu verbuchen hatte, schieden und scheiden sich die Geister. Doch selbst diejenigen, die zu einer kritischen Haltung tendieren, nehmen die Research \& Analysis (R\&A) Branch von dieser Kritik meist aus.

Wie eben dargelegt, war der Aufgabenbereich der R\&A-Branch in der von Präsident Roosevelt am 11.Juli unterzeichneten Order die einzige Funktion, zu der der COI ausdrücklich autorisiert war. Dem Gedanken, alle verfügbare Information in einer Zentrale zu sammeln, um sie von Experten aus Wissenschaft und Technik auswerten und zu Analysen verarbeiten zu lassen, die als Grundlage für politische Entscheidungen und strategische Planungen dienen konnten, kam in den von Donovan entwickelten Vorstellungen zudem zentrale Bedeutung zu. Dennoch beschränkte sich sein Beitrag zum Auf- und Ausbau der mit diesen Aufgaben befaßten R\&A-Abteilung letztlich auf die anfängliche Aufbauphase, denn selbst ein Mann von Donovans schier unerschöpflicher Energie konnte nicht in kürzester Zeit eine so komplexe Organisation wie einen zentralen Nachrichtendienst aus dem Nichts schaffen, wenn er nicht bereit war, Kompetenzen zu delegieren.

$\mathrm{Da}$ der Auswahl der ersten leitenden Mitarbeiter um so größere Bedeutung zukam, als auch für R\&A keine „Blaupause“ existierte und grundlegende Strukturen erst zu entwickeln waren, ersuchte Donovan Archibald MacLeish, den Leiter der Library of Congress, aus Mitarbeitern der Bibliothek, der National Archives, des American Council of Learned Societies sowie Vertretern verschiedener anderer Organisationen und Universitäten einen Ausschuß zu bilden, der Empfehlungen für die personelle 
Ausstattung der R\&A-Branch geben sollte ${ }^{33}$. Damit wurde der Grundstein zur Ausbildung eines der frühesten und prägendsten Charakteristika der R\&A-Branch gelegt der einzigartigen Zusammensetzung ihres Mitarbeiterstabes. Schon nach wenigen Monaten hätte R\&A den Vergleich mit den Fakultäten auch mancher renommierter Universitäten kaum zu scheuen brauchen, denn bekannte Historiker, Politikwissenschaftler, Soziologen, Wirtschaftswissenschaftler, Psychologen, Geographen und Sprachwissenschaftler von über 35 Universitäten hatten sich für die Mitarbeit in der Abteilung gewinnen lassen ${ }^{34}$.

Die Rekrutierung dieser ersten R\&A-Mitarbeiter erfolgte weitgehend innerhalb der Grenzen eines akademischen „old boys' network“, da die anfangs für die Arbeit in der Abteilung gewonnenen Wissenschaftler ihrerseits häufig von ihnen geschätzte Kollegen und Doktoranden oder Studenten als R\&A-Mitarbeiter gewannen. Insofern war es kein Zufall, wenn der Mitarbeiterstab der Abteilung anfänglich eine deutliche „Ivy League"-Prägung aufwies; doch wäre es kaum gerechtfertigt, dahinter politisches Kalkül zu vermuten. Denn diese Personalzusammensetzung hatte sich nicht nur aufgrund der eben erwähnten persönlichen Bekanntschaften herausgebildet, sondern spiegelte auch getreulich den zu dieser Zeit noch unangefochtenen Vorrang der alten etablierten Universitäten der Ostküste im Bereich der Geisteswissenschaften wider, wobei Harvard und Yale anfangs den größten Teil der R\&A-Mitarbeiter stellten ${ }^{35}$. Freilich dürfte sich diese „Ivy League“-Prägung insofern vorteilhaft für die Arbeit der Abteilung ausgewirkt haben, als die R\&A-Mitarbeiter gewohnt waren, in ähnlichen Kategorien zu denken wie die potentiellen Washingtoner Abnehmer der von ihnen ausgearbeiteten Studien und Analysen, die schließlich ebenfalls häufig an den Eliteuniversitäten der Ostküste studiert hatten ${ }^{36}$. Wie ein Großteil dieses „Kundenkreises“ waren viele Mitarbeiter der R\&A-Branch überdies deutlich vom liberalen Gedankengut und dem reformerischen Geist des New Deal geprägt.

Der Umstand, daß die Liste der R\&A-Mitarbeiter bald einem Who's Who der amerikanischen Geistes- und Sozialwissenschaften ähnelte, brachte Donovans Behörde von Anfang an einen gewissen Neid und reichlich Spott ein. Selbst in der nationalsozialistischen Propaganda wurde gehöhnt, der Mitarbeiterstab des neuen US-Nachrichtendienstes - dem die anfänglichen Notunterkünfte bald zu eng wurden und der sich deshalb ein eilig, aber nur unvollständig geräumtes Gebäude des National Health Institute mit dem dort zurückgebliebenen Experimentierzoo teilen mußte - bestehe aus "fifty professors, twenty monkeys, ten goats, twelve guinea pigs, and a staff of Jewish scribblers" ${ }^{37}$. Innerhalb des COI und in Washington wurde R\&A bald unter dem nicht immer respektvoll gemeinten Spitznamen "the Campus“ bekannt, denn in dem "un-

${ }^{33}$ OSS War Report I, S.48 f.; vgl. dazu und zum Folgenden auch Katz, Foreign Intelligence, S. $5 \mathrm{ff}$.

34 OSS War Report I, S.11; NA, RG 226, E 38, b 3 (Reading Files); zum „recruiting ground “ Universität Winks, Cloak, S. 23 ff., sowie Katz, a. a.O.

${ }^{35}$ Vgl. dazu Smith, Shadow Warriors, S.361 f.; und ausführlich Winks, Cloak, S.60ff.

${ }^{36}$ Smith, Shadow Warriors, S. 362; Kritik an der anfänglich nach rein akademischen Qualifikationen ausgerichteten Rekrutierung von Mitarbeitern in NA, RG 226, R\&A-History, S. $59 \mathrm{ff.}$

37 Zit. nach Ford, Donovan, S.122; vgl. auch Dunlop, Donovan, S.308; Troy, Donovan, S. 87; sowie Smith, OSS, S.1, der als einziger eine Quelle angibt: Drew Pearson, Washington Merry Go-Round, mit einer für den 3.12. 1941 vermerkten nationalsozialistischen Propagandasendung. 
usually pure academic ghetto ${ }^{\circ 38}$, das die R\&A-Branch bald darstellte, gediehen offenbar nicht nur viele der der akademischen Welt zugeschriebenen Tugenden, sondern wohl auch so manche der ihr eigenen Untugenden ${ }^{39}$.

Die Schlichtung der daraus resultierenden Probleme und Spannungen scheint denn auch das bereits Anfang August 1941 als R\&A-Führungsgremium eingerichtete Board of Analysts gelegentlich überfordert zu haben ${ }^{40}$. Unter Vorsitz des für die Leitung der R\&A-Branch gewonnenen Historikers James Phinney Baxter III sollte dieser Ausschuß die Arbeit der Abteilung planen und überwachen ${ }^{41}$; zu seinen Mitgliedern zählten mehrere Harvard-Professoren - der Historiker William Langer, der später Baxters Nachfolger werden sollte, der Wirtschaftswissenschaftler Edward S. Mason und Donald C. McKay, ein Spezialist für französische Geschichte - sowie der Politologe Joseph R. Hayden von der University of Michigan, der Wirtschaftswissenschaftler Calvin Hoover von der Duke University und der Militärhistoriker Edward Mead Earle vom Institute of Advanced Studies in Princeton. Später sollte dieser rein akademische Zirkel noch durch Vertreter des Außenministeriums sowie des Kriegs- und des Marineministeriums erweitert werden.

So beeindruckend personelle Besetzung wie Kompetenzbereich dieses bald mit dem Spitznamen „College of Cardinals" belegten Führungsgremiums auch waren, sollte es die ihm zugedachten Funktionen letztlich nie ganz ausfüllen. Ein Grund dafür war die Überlastung der Ausschußmitglieder, die alle noch vielerlei andere Verpflichtungen zu erfüllen hatten, wobei es sich anscheinend gelegentlich als besonders problematisch erwies, daß mehrere Ausschußmitglieder zugleich Leiter einer R\&A-Abteilung waren und deshalb zu einer gewissen Parteilichkeit neigten, die der Konsensfähigkeit erfordernden Arbeit dieses Gremiums nicht zuträglich war.

Immerhin war mit der Einsetzung des Board of Analysts Anfang August 1941 ein erster Schritt zum Aufbau der R\&A-Branch getan. Neben der Rekrutierung von Mitarbeitern gehörte es zunächst zu den vordringlichsten Aufgaben der Ausschußmitglieder, Vorstellungen darüber zu entwickeln, wie die Abteilung die ihr zugewiesenen Funktionen eigentlich erfüllen sollte. Der den in R\&A versammelten Akademikern vom OSS War Report zugeschriebene Ehrgeiz, einen Beitrag zur Entwicklung nachrichtendienstlicher Arbeitsweisen zu leisten ${ }^{42}$, drückte sich dabei in dem für R\&A so charakteristischen Bemühen aus, stets wissenschaftlich und systematisch vorzugehen: „the basis of the research and analysis function was conceived to be the collection of all evidence relevant to the subject at hand and the application to that evidence of the inductive and deductive processes of analysis" ${ }^{43}$. Wie in der R\&A-History betont

${ }^{38}$ Smith, Shadow Warriors, S.362.

39 Vgl. dazu die farbige Darstellung des OSS- bzw. R\&A-Mitarbeiters Alcorn, No Bugles for Spies, S.71-93.

40 Dazu Corson, Armies of Ignorance, S.172.

${ }^{41}$ OSS War Report I, S.50; zum Folgenden auch Katz, Foreign Intelligence, S. $4 \mathrm{ff}$.; Winks, Cloak, S.70f., und Troy, Donovan, S. 84 f. Die Sitzungen des Ausschusses sind dokumentiert in NA, RG 226, E 58: Minutes of the Meetings of the Board of Analysts, 1941-43. Der Ausschuß wurde im Dezember 1943 aufgelöst; seine Funktionen wurden im wesentlichen vom Projects Committee übernommen, siehe dazu unten III., 2. Teil.

42 OSS War Report I, S. 13; vgl. auch Katz, Foreign Intelligence, S.6f.

${ }^{43}$ NA, RG 226, R\&A-History, (II. The Nature of Research and Analysis Activities), S. 4. 
wird, war man sich zwar bewußt, damit nur die Beachtung altehrwürdiger Grundsätze solider Wissenschaft zu fordern, doch glaubte man feststellen zu müssen, daß dieses Vorgehen für die amerikanischen Nachrichtendienste „surely and shamefully new in practice" sei ${ }^{44}$. Daß man sich in R\&A in so hohem Maße einem Ideal strenger Wissenschaftlichkeit verpflichtet fühlte und damit auch die Forderung nach „absoluter“ Objektivität und einer entsprechend sachlichen, nüchtern-emotionslosen Darstellungsweise verband, spiegelte nicht zuletzt die zeitgenössische Diskussion um eine „Verwissenschaftlichung" der Geisteswissenschaften wider ${ }^{45}$.

Um R\&A mit dem Material zu versorgen, das die Mitarbeiter wissenschaftlich auswerten sollten, war es nach Donovans Ansicht nicht notwendig, zuerst eine eigens mit der Sammlung von Daten und Informationen befaßte Abteilung aufzubauen. Militärische Stellen sowie Außenministerium, FBI und einige andere Regierungsbehörden verfügten schließlich bereits über entsprechende Abteilungen, und offenbar war es Donovan ernst mit seiner Absicht, nicht in deren Kompetenzen einzugreifen, sondern sich auf die ihm zugewiesene Aufgabe der „Koordination“ der von diesen Stellen gesammelten Informationen zu beschränken. Die für die wissenschaftliche Analyse und Aufbereitung dieser Materialien benötigten Hintergrundinformationen waren nach Donovans Ansicht aus Büchern, Fachzeitschriften und Zeitungen zu gewinnen. Donovan war deshalb daran gelegen sicherzustellen, daß R\&A die reichhaltigen Ressourcen der Library of Congress nutzen konnte, und in Gesprächen mit Archibald MacLeish hatte er bereits im Juni die Grundlage für eine Zusammenarbeit zwischen der Bibliothek und der R\&A-Branch geschaffen ${ }^{46}$.

Aufgrund der zwischen Donovan und MacLeish getroffenen Vereinbarungen konnten die Mitarbeiter der im August 1941 eingerichteten Division of Special Information (DSI) so zunächst in der Library of Congress ein Unterkommen finden ${ }^{47}$. Die Hauptaufgabe dieser nach regionalen Schwerpunkten untergliederten R\&A-Abteilung bestand darin, den von Donovan für die Analyse aktueller Informationen als so wichtig erachteten Grundstock an "background knowledge“ zusammenzutragen. Neben den Ressourcen der Library of Congress zogen die DSI-Mitarbeiter dafür auch Bestände anderer Bibliotheken heran und werteten in Firmen- und Bankarchiven verfügbare Materialien aus.

Leiter der DSI war der renommierte Historiker William L. Langer, der im Sommer 1942 als Nachfolger des offiziell aus gesundheitlichen Gründen zurückgetretenen Baxter auch die Leitung der R\&A-Branch übernehmen sollte ${ }^{48}$. Der 1896 als Kind deutscher Einwanderer in Boston geborene Langer war in relativ ärmlichen Verhältnissen aufgewachsen; dank seiner Begabung und eines offenbar schon in jungen Jahren entwickelten Ehrgeizes gelang es ihm jedoch, mit Hilfe eines Stipendiums in Harvard moderne Sprachen zu studieren, um Lehrer für Deutsch und Französisch zu werden ${ }^{49}$. Da

\footnotetext{
44 NA, RG 226, R\&A-History, a.a.O.

${ }^{45}$ Etwas spöttisch dazu Smith, Shadow Warriors, S.362f.; vgl. auch Katz, Foreign Intelligence, S. $15 \mathrm{ff}$.

${ }^{46}$ OSS War Report I, S.9.

47 Vgl. zum Folgenden OSS War Report I, S.53.

${ }^{48}$ Langer hatte ursprünglich wohl angenommen, daß er von Anfang an die Leitung der R\&ABranch übernehmen würde; dazu ausführlicher in III., 2. Teil.

49 Zum Folgenden Langers Autobiographie, Ivory Tower.
} 
Langer das auf vier Jahre angelegte Studienprogramm in nur drei Jahren absolvierte, konnte er seine erste Stellung bereits 1915 antreten. Von seiner Arbeit als Deutschlehrer in einem Internat in keiner Weise ausgefüllt, begann Langer in seiner Freizeit bald, sein schon immer waches Interesse für Geschichte und Politik zu vertiefen, und nachdem er im Ersten Weltkrieg als Freiwilliger Militärdienst geleistet hatte, entschloß er sich nach Kriegsende, nicht zu seiner Lehrertätigkeit zurückzukehren, sondern in Harvard ein Promotionsstudium in Geschichte aufzunehmen.

In seiner Dissertation beschäftigte sich Langer mit der französisch-russischen Allianz zwischen 1891-1894, einem für amerikanische Verhältnisse damals eher exotischen Thema. Nach einem fast einjährigen Forschungsaufenthalt in Europa und dem Abschluß des Promotionsverfahrens begann Langer, an der Clark University in Worcester europäische Geschichte zu lehren und daneben bei der eben erst gegründeten Zeitschrift Foreign Affairs mitzuarbeiten ${ }^{50}$. Mit seiner fachlichen Orientierung bewies Langer ein für amerikanische Geisteswissenschaftler jener Zeit relativ ungewöhnliches Interesse an europäischer Zeitgeschichte. Seine Expertise auf diesem Gebiet fand Anerkennung, als er auf den Coolidge-Lehrstuhl nach Harvard berufen wurde, und nachdem schon seine 1929 publizierte Dissertation ausgezeichnete Rezensionen erhalten hatte, avancierten seine 1931 bzw. 1935 erscheinenden Darstellungen der European Alliances and Alignments sowie der Diplomacy of Imperialism bald zu Klassikern. In diesen umfang- und detailreichen Werken demonstrierte Langer seine vollständige Beherrschung der klassischen Methoden Rankescher Diplomatiegeschichte, wobei sein Interesse vorrangig der Frage nach den Bedingungen internationaler Stabilität galt. Angesichts der politischen und gesellschaftlichen Entwicklungen in Europa begann Langer aber auch, die Grenzen der Rankeschen Methoden zu erkennen und die Notwendigkeit sozialgeschichtlicher Fragestellungen und Arbeitsweisen zu konzedieren.

In den dreißiger Jahren blieb Langer ein aufmerksamer Beobachter des europäischen Zeitgeschehens, wobei er stets bemüht war, die politischen Entwicklungen vor ihrem geschichtlichen Hintergrund zu sehen. Illustrativ ist in diesem Zusammenhang ein von ihm Anfang 1938 verfaßter Artikel mit dem Titel When German Dreams Come True $^{51}$, in dem er das von Hitler in Mein Kampf formulierte Gedankengut als Erbe der im 19. Jahrhundert entwickelten nationalistischen, sozialdarwinistischen und imperialistischen Vorstellungen verstanden wissen wollte. Langer beschränkte sich jedoch nicht auf diese Analyse, sondern versuchte, davon ausgehend auf die künftige deutsche Politik zu schließen. Seiner Ansicht nach strebten die Deutschen danach, einen mitteleuropäischen Machtblock zu errichten, der das nach dem Verschwinden der österreichisch-ungarischen Donaumonarchie entstandene Vakuum füllen würde. Doch, wie Langer meinte: "The new bloc would, undoubtedly, be more inclusive than the old, and the German hegemony would be far more effective." Die Annexion Österreichs sei nur der erste Schritt auf dem Wege zur Verwirklichung dieses „age-old urge and [...] age-old dream"; ganz richtig sagte Langer in diesem Zusammenhang auch die Besetzung der Tschechoslowakei voraus. Weder Großbritannien noch Frankreich, ge-

\footnotetext{
${ }^{50} \mathrm{Zu}$ Foreign Affairs, dem Organ des 1921 begründeten Council on Foreign Relations, siehe Wala, Winning the Peace, S. $33 \mathrm{ff}$.

51 Abgedruckt in Langer, Explorations in Crisis, S. 227-243.
} 
schweige denn Rußland würden nach Langers Analyse in der Lage bzw. willens sein, den Deutschen Einhalt zu gebieten; doch spätestens wenn dieses neue Deutsche Reich versuchen würde, Kolonialpolitik zu betreiben, würden die Briten erkennen müssen, „that they have been living in a fool's paradise, for the new Greater Germany promises to be infinitely stronger than the antagonist of 1914 ".

In diesem Artikel hatte Langer die Arbeitsweise der R\&A-Branch im Grunde vorweggenommen und vor allem auch die später von Donovan vertretene Auffassung bestätigt, daß schon allein die sorgfältige Analyse frei verfügbarer Materialien durch Experten zu aufschlußreichen Erkenntnissen verhelfen konnte. So gehörte die Ausarbeitung derartiger Analysen denn auch zu den Hauptaufgaben der nach regionalen Schwerpunkten untergliederten DSI, wobei das Schwergewicht - dem Kriegsgeschehen entsprechend - zunächst auf Europa lag. Allein drei DSI-Abteilungen widmeten sich unter Leitung hervorragender Gelehrter dem Studium der dortigen Entwicklungen: Die Central Europe Section unterstand Walter L. Dorn, der zuvor als Professor für moderne europäische Geschichte bzw. als Spezialist für preußische Geschichte an verschiedenen amerikanischen Universitäten gelehrt hatte und der später als Berater für die Planung und Ausführung der amerikanischen Deutschlandpolitik fungieren sollte $^{52}$. Die nächste Wissenschaftlergeneration war ebenfalls schon vertreten, repräsentiert etwa durch Carl E. Schorske, einem der wenigen Langer-Schüler, zu denen der häufig als verschlossen beschriebene Langer wohl auch eine persönliche Beziehung hatte. Schorske sollte sich später als Autorität auf dem Gebiet europäischer Geistesgeschichte einen Namen machen, und unter seinen Veröffentlichungen finden sich auch mehrere Studien zur deutschen Zeitgeschichte ${ }^{53}$.

Neben der Central Europe Section umfaßte die DSI noch die unter der Leitung des bekannten Politologen Robert K. Gooch stehende Western Europe Section sowie die Eastern Europe Section, in der mit Mitarbeitern wie etwa dem Historiker Robert L. Wolff, der später in Harvard osteuropäische Geschichte lehrte, ebenfalls ausgezeichnete Fachleute beschäftigt waren. Die unter der Leitung des Yale-Historikers Sherman Kent stehende Mediterranean and Africa Section hatte als erste DSI-Abteilung Gelegenheit, ihre Leistungsfähigkeit mit ihren in Vorbereitung für die Operation TORCH ausgearbeiteten Nordafrika-Studien unter Beweis zu stellen und dabei auch „, the first victory' for R\&A's methodology “54 zu erringen. Mit seiner Arbeit als Leiter der Mediterranean and Africa Section bzw. der späteren Europe-Africa Division legte Sherman Kent den Grundstein für seine Nachkriegskarriere als Direktor des von William Lan-

52 Directory of American Scholars 1951, S. 237. Kritik an Dorn in NA, RG 226, R\&A-History, (IV.), S.76; vgl. dazu auch Söllner (Hrsg.), Archäologie der Demokratie, Bd.2, S. 23. Siehe auBerdem das von Niethammer gezeichnete Persönlichkeitsbild Dorns in: Dorn, Inspektionsreisen in der US-Zone, S. 8 ff. Von der Arbeit der Central Europe Section während der Aufbauphase des COI ist heute nicht mehr viel zu identifizieren, was sich zum Teil aus der ursprünglichen Funktion der DSI als eine Art von Dienstleistungsstelle innerhalb der R\&A-Branch erklären mag; vgl. NA, RG 226, E 58, b 1, Book I, Exhibit No.3.

53 Directory of American Scholars 1951, S. 832. Schorskes spätere Publikationen umfassen u.a.: The Problem of Germany, New York 1947, German Social Democracy, 1905-1917: The Development of the Great Schism, Cambridge 1955; Explorations in Crisis, eine 1969 von Schorske als Festschrift herausgegebene Sammlung von Langers Aufsätzen.

54 Donovan zit. nach Winks, Cloak, S. 85; zur COI/OSS-Beteiligung an TORCH siehe III., 1. Teil. 
ger innerhalb der CIA aufgebauten Office of National Estimates (ONE) ${ }^{55}$, und neben Langer entwickelte sich Kent zur prägenden Persönlichkeit innerhalb der R\&ABranch.

Nachdem mit der Einrichtung der DSI ein wesentliches Element von Donovans Konzept verwirklicht war, wurde Anfang September mit der Central Information Division (CID) ein weiterer wichtiger 'Teil seiner Vorstellungen realisiert. Aufgabe der

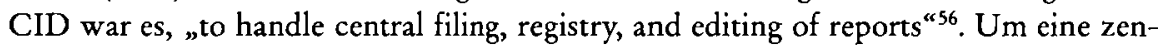
trale Kartei aufzubauen, mußten sich die CID-Mitarbeiter allerdings erst einmal die Materialien beschaffen, die sie katalogisieren sollten. Schon dieser erste Schritt ihrer Arbeit erwies sich freilich als alles andere als einfach, denn nach Darstellung der R\&A-History war es nur durch "cajolery, bullying, logic and semi-theft " möglich, eine Sammlung aufzubauen, die anfänglich kaum mehr enthielt als „quarterly price reports, Axis orders of battle, estimated requirements of the British Empire, and other such luke-warm intelligence as the cstablished agencies would release to a new broth-

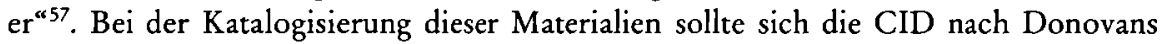
Vorstellungen dann nicht etwa darauf beschränken, das altehrwürdige "file and forget“-System der meisten Regierungsbehörden einmal mehr anzuwenden. Donovan bestand vielmehr darauf, daß der COI über das modernste und effizienteste Katalogisierungssystem verfügen sollte ${ }^{58}$. Daß der COI bekam, was Donovan wollte, bezeugt noch heute der in den Washingtoner National Archives aufbewahrte, fast eine Million Karten umfassende Index, der wahrlich das „institutional ,memory “659 des COI bzw. OSS darstellte.

Dieser Index war das Werk von Wilmarth Sheldon Lewis ${ }^{60}$, einem aus wohlhabender Familie stammenden Yale-Absolventen, den Archibald MacLeish im August 1941 für die Mitarbeit beim COI gewonnen hatte. Den leidenschaftlichen Literaturliebhaber Lewis faszinierte die Frage, wie die im COI anzusammelnde Vielzahl von Einzelinformationen und Daten am besten zu organisieren wäre. $\mathrm{Daß}$ er dieses Problem mit Bravour löste, trug unschätzbar viel zur Effizienz und Qualität der Arbeit von R\&A bei, denn dank eines ausgefeilten Systems von Querverweisen erlaubte der von Lewis angelegte Index schon lange vor unserem Computerzeitalter einen schnellen und sicheren Zugriff auf vorhandene Daten und Informationen, so daß angeblich sogar das State Department gelegentlich bei R\&A anfragte, über welche Konsularberichte es selbst eigentlich verfüge $\mathrm{e}^{61}$.

Im Herbst 1941 wurden neben der nach regionalen Schwerpunkten gegliederten DSI drei funktionale, d.h. fachlich spezialisierte R\&A-Abteilungen eingerichtet. Bereits im September wurde mit dem Aufbau der Psychology Division begonnen ${ }^{62}$.

55 Vgl. auch Kent, Strategic Intelligence for American World Policy, Princeton 1949.

56 OSS War Report I, S. 49, siehe auch S.60 und 169.

57 NA, RG 226, R\&A-History, (CID), S.4.

58 OSS War Report I, S. 49, 60.

59 Corson, Armies of Ignorance, S. 171.

${ }^{60}$ Zum Folgenden Winks, Cloak, S. $96 \mathrm{ff}$.

${ }_{61}$ Ebenda, S. 100; vgl. auch NA, RG 226, R\&A-History, (CID), S.14f.

62 Zum Folgenden OSS War Report I, S.59f.; die Psychology Division wurde allerdings im Rahmen der im Januar 1943 durchgeführten Reorganisation der R\&A-Branch aufgelöst; dazu unten III., 2. Teil. 
Die Mitarbeiter dieser zur damaligen Zeit im Rahmen eines Geheimdienstes zweifellos exotisch anmutenden Abteilung sollten einerseits ihre Expertise bei der Analyse nationalsozialistischer Propaganda einbringen ${ }^{63}$; andererseits sollten sie bei der Planung amerikanischer Propaganda mitwirken, was enge Zusammenarbeit mit dem unter Leitung von Robert E. Sherwood stehenden Foreign Information Service (FIS) erforderte. Der FIS hatte als erste COI-Abteilung schon im August 1941 seine Arbeit aufgenommen und konnte mit seinem ab Februar 1942 ausgestrahlten Programm der „Voice of America“ auch schon früh Leistungen vorweisen. Aufgrund schwerwiegender Differenzen zwischen Donovan, der Propaganda als integralen Bestandteil psychologischer Kriegführung verstanden wissen wollte, und Sherwood, der - wie viele der von ihm rekrutierten liberal-demokratischen, dem New Deal verpflichteten FISMitarbeiter - eher politische "Aufklärungsarbeit" leisten wollte, erwies sich der FIS aber auch als die problematischste COI-Abteilung. Letztlich sollten die Differenzen zwischen Donovan und Sherwood zur Ausgliederung des FIS aus dem COI und seiner Übernahme durch das im Juni 1942 gegründete Office of War Information füh$\operatorname{ren}^{64}$.

Als weitere funktionale Abteilung wurde im Oktober die Economics Division unter Leitung des Wirtschaftswissenschaftlers Edward S. Mason begründet ${ }^{65}$. Sie gliederte sich anfänglich nach den Arbeitsbereichen Agriculture and Standard of Living, Military Supplies, Labor Supply und Industrial Resources; später wurden noch einige andere Abteilungen gebildet. Die Mitarbeiter der Economics Division - darunter so bekannte Wissenschaftler wie Emile Despres, Calvin Hoover, Charles Kindleberger und Wassily Leontief - zeigten von Anfang an großes Engagement, und schon ihre erste umfassende Studie, „The German Economic and Military Position“ vom 12. Dezember $1941^{66}$, war methodisch wie inhaltlich insofern ein Glanzstück, als in ihr unter Ausschöpfung aller erdenklicher Informationsquellen Ergebnisse erarbeitet und Voraussagen getroffen wurden, die zwar manchen gängigen Ansichten widersprachen, sich aber letztlich als zutreffend erweisen sollten ${ }^{67}$.

Ebenfalls im Oktober 1941 wurde mit dem Aufbau der Geographic Division begonnen ${ }^{68}$; ihre Mitarbeiter fertigten Landkarten als Illustration zu R\&A-Berichten an, steliten Informationen über Infrastruktur, Hafenanlagen und Stadtpläne zusammen

${ }^{63}$ Z.B. NA, RG 59, R\&A 608, „Design of Propaganda in German Foreign Newsreels (,Auslandstonwochenschau')“, 19.3. 1942; NA, RG 59, R\&A 616, „The Radio Propaganda Atmosphere of the German Public “, 21.3.1942; ausführlicher dazu unten II., 2. Teil.

${ }^{64} \mathrm{Zu}$ den Differenzen zwischen Donovan und Sherwood, zur Arbeit des FIS und in diesem Zusammenhang auch zur Problematik des Einsatzes von Propaganda OSS War Report I, S.10f., 31-48. Zur Geschichte des OWI Winkler, Politics of Propaganda.

${ }^{65}$ Zum Folgenden OSS War Report I, S. 56 ff.; NA, RG 226, R\&A-History, (IV. Europe-Africa Division), S.3ff.

${ }^{66}$ NA, RG 59, R\&A 214 A, B; vgl. auch NA, RG 226, R\&A-History, (IV. Europe-Africa Division), S. $4 \mathrm{ff}$. Diese Studie war die erste umfassende R\&A-Arbeit, die Roosevelt vorgelegt wurde, vgl. FDRL, PSF/Subject/OSS, b 163, f 1, sowie b 165, f 7 (Langer an Roosevelt, 10.3. 1942); ausführlich dazu unten II., 1. Teil.

${ }^{67}$ Vgl. dazu auch OSS War Report I, S. $56 \mathrm{ff}$;; weitere Beispiele zur Arbeitsweise der Economics Division bei Winks, Cloak, S.86ff. Die R\&A-History enwähnt aber auch Fehleinschätzungen der deutschen Wirtschaftskapazität durch die Economics Division, dazu unten II., 1. Teil.

${ }^{68}$ Zum Folgenden OSS War Report I, S. 58f.; vgl. auch Winks, Cloak, S. $89 \mathrm{f}$. 
und analysierten die Genauigkeit und Aussagekraft veröffentlichter kartographischer Materialien.

Problematisch an dieser ursprünglichen Struktur der Research and Analysis Branch war, daß die drei funktionalen Abteilungen, also die Psychology, Economics und Geographics Divisions, nicht etwa mit der nach regionalen Schwerpunkten gegliederten Division of Special Information zusammenarbeiteten, sondern vielmehr direkt der Leitung des Board of Analysts unterstanden. Dies führte zu Überschneidungen, die Verschwendung von Arbeitskraft bedeuteten und zu Spannungen führten, wobei die Beziehungen zwischen der Economics Division und der Eastern Europe Section der DSI besonders beeinträchtigt waren. Für eine Organisation, die geschaffen worden war, um eine bessere Koordination der amerikanischen Nachrichtendiensttätigkeit zu gewährleisten, war dies ein schwerwiegender Minuspunkt, zumal diese Probleme erst Anfang 1943 im Zuge einer grundlegenden Reorganisation der R\&A-Branch gelöst wurden ${ }^{69}$. Dennoch war mit den soeben beschriebenen Abteilungen bis Ende 1941 eine arbeitsfähige Grundstruktur geschaffen worden. In dieser Aufbauphase kam der R\&A-Branch zweifellos zugute, daß ihr Aufgabenbereich die einzige Funktion war, zu der Donovans neue Behörde in der präsidentiellen Order vom 11. Juli ausdrücklich autorisiert worden war. Trotzdem mußte sich R\&A erst einmal einen "Kundenkreis“ schaffen, wobei die Abteilung - wie der ganze COI - angesichts des innerhalb der Washingtoner Bürokratie herrschenden Kampfes um Mittel, Macht und Einfluß unter großem Leistungsdruck stand.

Um potentielle Interessenten in den Washingtoner Regierungsbehörden mit der Arbeit von R\&A in Berührung zu bringen, verfiel DSI-Chef Langer auf den Gedanken, in einer wöchentlich erscheinenden Publikation einen Überblick über den Fortgang und die Ergebnisse von R\&A-Studien zu geben ${ }^{70}$. Als „SECRET“ klassifiziert, fand diese Publikation unter dem Titel The War This Week ab Anfang Dezember 1941 in Washingtoner Regierungskreisen relativ weite Verbreitung. Obwohl über die Vergabe der einzelnen numerierten Exemplare Buch geführt wurde, fürchtete man in militärischen Kreisen wohl nicht ganz zu Unrecht, daß auf diese Weise klassifizierte Information nur allzu leicht in die Hände von Unbefugten geraten könne. So sah sich Donovan schließlich veranlaßt, die Einstellung dieser Publikation anzuordnen, was er angeblich mit dem vielzitierten Kommentar tat, Akademiker seien „like Chorus girls, who have beautiful legs and like to show them ${ }^{\text {"71 }}$.

Aber auch Donovan hatte sich schon früh Gedanken darüber gemacht, wie die Ergebnisse der Arbeit seiner Organisation am besten darzustellen und weiterzuvermitteln wären. Als Anwalt hatte er gelernt, wie wichtig es war, große Mengen komplexer Daten und Informationen in eingängiger Weise zu präsentieren, und er hatte seine diesbezüglichen Fähigkeiten in zahlreichen Prozessen unter Beweis gestellt. Seiner Auffassung nach mußte dies auch Teil seiner Aufgabe als Coordinator of Information sein, denn die besten Studien und Analysen nützten schließlich wenig, wenn sie auf den sowieso stets überladenen Schreibtischen der Entscheidungsträger untergingen. Vor diesem Hintergrund entwickelte Donovan zusammen mit dem erfolgreichen Holly-

${ }^{69}$ Siehe dazu III., 2. Teil.

70 Dazu und zum Folgenden OSS War Report I, S.51; Winks, Cloak, S.75.

${ }^{71}$ Hier zit. nach Winks, Cloak, S. 75. 
wood-Regisseur Merian C. Cooper und einem Ausschuß von Filmexperten, Technikern und Elektronikfachleuten ehrgeizige Pläne für eine Visual Presentation Branch $(V P)^{72}$, die beispielsweise über ein mit der neuesten Technologie ausgestattetes Gebäude verfügen sollte, in dessen Räumen die verschiedenen Aspekte der internationalen Situation mit Hilfe ausgefeilter Präsentationstechniken leicht faßbar dargestellt werden könnten. Obwohl Donovan seine grandiosen Pläne aus finanziellen Gründen erheblich modifizieren mußte, umfaßte die VP Branch schon bald eine Field Photographic Division, die den amerikanischen "war effort" photographisch und filmisch dokumentierte, sowie die - später von R\&A übernommene - Pictorial Records Section, deren Aufgabe die Sammlung und Auswertung von photographischen und filmischen Materialien war $^{73}$, und schließlich die Graphic Section, die herangezogen wurde, wenn es galt, Ergebnisse von R\&A-Studien in besonderer Weise darzustellen ${ }^{74}$.

Donovans Pläne für die VP Branch illustrieren ebenso wie manche andere Vorstellungen, die er beim Aufbau des COI entwickelte, in welch hohem Maße er stets neuen, den traditionellen Rahmen sprengenden Ideen gegenüber aufgeschlossen war. Doch so schnell seine Begeisterung für ein neues Projekt auch entflammt war, so schnell konnte sie auf ein wieder neues übergehen. Die R\&A-Branch bekam die Auswirkungen dieser Sprunghaftigkeit in mehrfacher Hinsicht zu spüren. Vor allem in der Anfangsphase des COI, als Donovan in besonderem Maße daran gelegen sein mußte, Roosevelt und andere potentielle Interessenten mit den vom COI erbrachten Leistungen zu beeindrucken, mußten R\&A-Mitarbeiter stets damit rechnen, immer neue, stets überaus dringliche Anfragen und Arbeitsaufträge von Donovan zu erhalten ${ }^{75}$. Doch obwohl die von R\&A ausgearbeiteten Studien und Analysen für geraume Zeit das einzige waren, was der COI an konkreten Leistungen überhaupt vorzuweisen hat$\mathrm{te}^{76}$, verlor Donovan in gewisser Weise das Interesse an dieser von ihm ursprünglich als so wichtig erachteten Abteilung. Das bedeutete zwar keineswegs, daß er die Arbeit der R\&A-Branch plötzlich geringschätzte, doch der Aufbau anderer COI-Abteilungen nahm seine Aufmerksamkeit in Anspruch, und im Vergleich mit den Aufgaben einer Spionage- oder Sabotageabteilung, wie sie der COI bald umfassen sollte, fehlte es der Arbeit von R\&A zweifellos an "drama and excitement"77. Auf lange Sicht sollte sich allerdings zeigen, daß die R\&A-Wissenschaftler mit ihren Karteikarten über die vielleicht „most powerful weapon“ im Arsenal der Geheimdienstwaffen verfügten ${ }^{78}$.

72 Dazu OSS War Report I, S.12f., 67 f.; Troy, Donovan, S. $108 f$.

73 Zur Arbeit dieser Abteilung Winks, Cloak, S. $106 \mathrm{f}$.

74 Dazu OSS War Report I, S. 13.

${ }^{75}$ Ebenda, S.61, sowie Winks, Cloak, S. $67 \mathrm{f}$.

${ }^{6} \mathrm{Vgl}$. dazu auch die Beispiele in OSS War Report I, S.17; siehe außerdem die von Cline, Secrets, S.48 entworfene Graphik zur Organisation des COI.

77 Langer, Ivory Tower, S.187. Das COI-Budget spiegelt diese veränderte Gewichtung deutlich wider, vgl. OSS War Report I, S.13; sowie die bei Troy, Donovan, S.112 abgedruckte "Summary of 1942 Budget Request “ des COI. Vgl. auch NA, RG 226, R\&A-History, (III.), S. 23: „Donovan, though generally sympathetic toward R\&A and possessed of considerable respect for the total of its talents, was fundamentally more interested in operations than in intelligence." Zur Gewichtung von „intelligence“ und „operations“ grundsätzlich Laqueur, World of Secrets, S. 32, sowie Ameringer, U.S. Foreign Intelligence, S. 396-405.

${ }^{78}$ So Winks, Cloak, S. 63; ähnlich Smith, Shadow Warriors, S. 361. 
